\title{
Non-degenerate magnetic alignment of self-assembled mesophases
}

\author{
Paweł W. Majewski* Chinedum O. Osuji ${ }^{\dagger}$
}

July 22, 2009

We present a method, which we call "rotational annealing", for the production of uniquely defined (non-degenerate) magnetic alignments of self-assembled mesophases and demonstrate its effectiveness for realizing near single-crystal quality materials over relatively short timescales.

Self-assembled block copolymer and surfactant mesophases present rich opportunities for the design of a variety of functional systems. Spatially uniform arrangement of the spherical, rod and sheet-like mesostructures formed by these chemically diverse materials offers truly exceptional advances in applications ranging from photovoltaics ${ }^{1}$ and photonic crystals $^{2-4}$ to high-density storage media ${ }^{5,6}$ and templates for materials synthesis. ${ }^{7-9}$ In many cases, a critical limitation in the realization of these utilities is the inability to reliably and precisely manipulate the microstructure of these materials. Considerable efforts have been devoted to understanding the mechanisms via which self-assembly or self-assembled structures in soft materials may be driven by external inputs, as recently reviewed for block copolymers. ${ }^{10}$

High magnetic fields ( $\gtrsim 2$ Tesla) offer promise as a flexible tool for nanostructure alignment in these systems. They circumvent the contact and dielectric breakdown issues associated with electric field alignment. ${ }^{11-14}$ Magnetic fields can be conveniently applied over macroscopic length scales $(>10 \mathrm{~cm})$ and to geometries or devices that are ill-suited to the use of other alignment techniques, such as flow fields or surface patterning. These include, for example, thin film coatings of large area substrates and coatings on recessed surfaces. The need for high fields and sufficiently large magnetic anisotropy in the system present some limitations to the utility of this approach in diamagnetic materials such as conventional polymers and surfactants. More importantly, field driven alignment in these systems generally results in the production of degenerate orientations of the phases, as discussed in Figure Fig. 1. While diamagnetic alignment of the hexagonal (HEX) phase of ionic surfactants such as sodium dodecyl sulphate (SDS) and cetyltrimethyl ammonium bromide (CTAB) has been demonstrated, ${ }^{15-18}$ the alignment of non-ionic surfactants has been more elusive. Thus far, it has only been reported at 30 Tesla as an intermediate step in the evaporative synthesis of mesoporous silica utilizing the hexagonal phase. ${ }^{19}$ By contrast, the production of macroscopically unique alignments of lamellar (LAM)

\footnotetext{
*Department of Chemical Engineering, Yale University, New Haven CT 06511

${ }^{\dagger}$ Department of Chemical Engineering, Yale University, New Haven CT 06511 E-mail: chinedum.osuji@yale.edu
} 
phases of either ionic or non-ionic surfactants has not been reported. As explained in Figure Fig. 1, it cannot be simply attained in surfactant systems due to a combination of the surfactant orientation at the inter-materials dividing surface (IMDS) and the negative diamagnetic anisotropy of alkyl chains, $\Delta \chi<0$.

Here, we report on a novel application of magnetic fields which can be used to align these materials in a facile manner. We demonstrate that a simple scheme which we term "rotational annealing," breaks the degeneracy of the diamagnetic alignment of the lamellar phase. This enables the systematic production of near single-crystal quality materials on relatively short timescales. We work with mesophases produced by non-ionic surfactants and show that both lamellar and hexagonal phases can be successfully field aligned. Our result is of particular significance as it describes a method for arriving at well defined morphologies in arbitrary geometries while circumventing any inherent magnetic degeneracy that may be present in the material. The use of non-ionic mesophases is noteworthy as they present more inert, and thus more convenient systems than their ionic counterparts for the templated synthesis of nanostructured materials.

Magnetic alignment of nanostructures relies on the presence of sufficient anisotropy in the magnetic susceptibility of the material at the nanoscale. The required anisotropy, essentially absent in amorphous organic materials, may be provided by the presence of liquid crystalline (LC) order in the material. In this manner, diamagnetic alignment of aqueous lyotropic surfactant phases, ${ }^{15,17-19}$ thermotropic small molecule systems ${ }^{20,21}$ thermotropic liquid crystalline block copolymers in the melt state ${ }^{22-25}$ and a semicrystalline block copolymer. ${ }^{26}$ have been advanced. In both block copolymer melts and lyotropic surfactant mesophases, only the uniaxial phases can be field aligned. That is, only the lamellar and hexagonal phases. The cubic bicontinuous and micellar phases are magnetically isotropic and thus not field responsive. The free-energy difference per unit volume due to the interaction of a magnetically anisotropic system with a field is given by Equation 1, where $\chi$ is the magnetic susceptibility, $\mathbf{B}$ is the magnetic field strength and $\theta$ is the angle between the field and the vector characterizing the orientation of the system, for example, the director in an LC system. Because diamagnetic materials repel field lines $(\chi<0)$, for positive anisotropy $\Delta \chi>0$ they align with their long axis (identified with $\chi_{\|}$) parallel to the field.

$$
\begin{aligned}
\Delta \chi & =\chi_{\|}-\chi_{\perp} \\
F(\theta, \mathbf{B}) & =-\frac{1}{3} \Delta \chi \mathbf{B}^{2} \frac{1}{2}\left\langle 3 \cos ^{2} \theta-1\right\rangle
\end{aligned}
$$

The degeneracy exhibited by block copolymers and surfactant mesophases is determined by the orientation of the relevant magnetically anisotropic moieties relative to the IMDS that defines the periodic mesostructures in the system. In liquid crystalline block copolymers for example, the mesogens in the material may have homogeneous (parallel to the surface) or homeotropic (perpendicular to the surface) anchoring at the IMDS. ${ }^{27}$ In lyotropic surfactant 
mesophases, the individual surfactant chains that give rise to the magnetic anisotropy in the system are by default oriented perpendicularly to the IMDS. For n-alkyl type surfactants, the anisotropy of the molecule is in general negative due to the large negative contribution from the hydrocarbon chain. ${ }^{28}$ As a result, application of a magnetic field drives alignment of hexagonally packed cylindrical domains uniquely, with their long axis along the field direction, but produces a degenerate arrangement of lamellar microdomains, Figure Fig. 1.

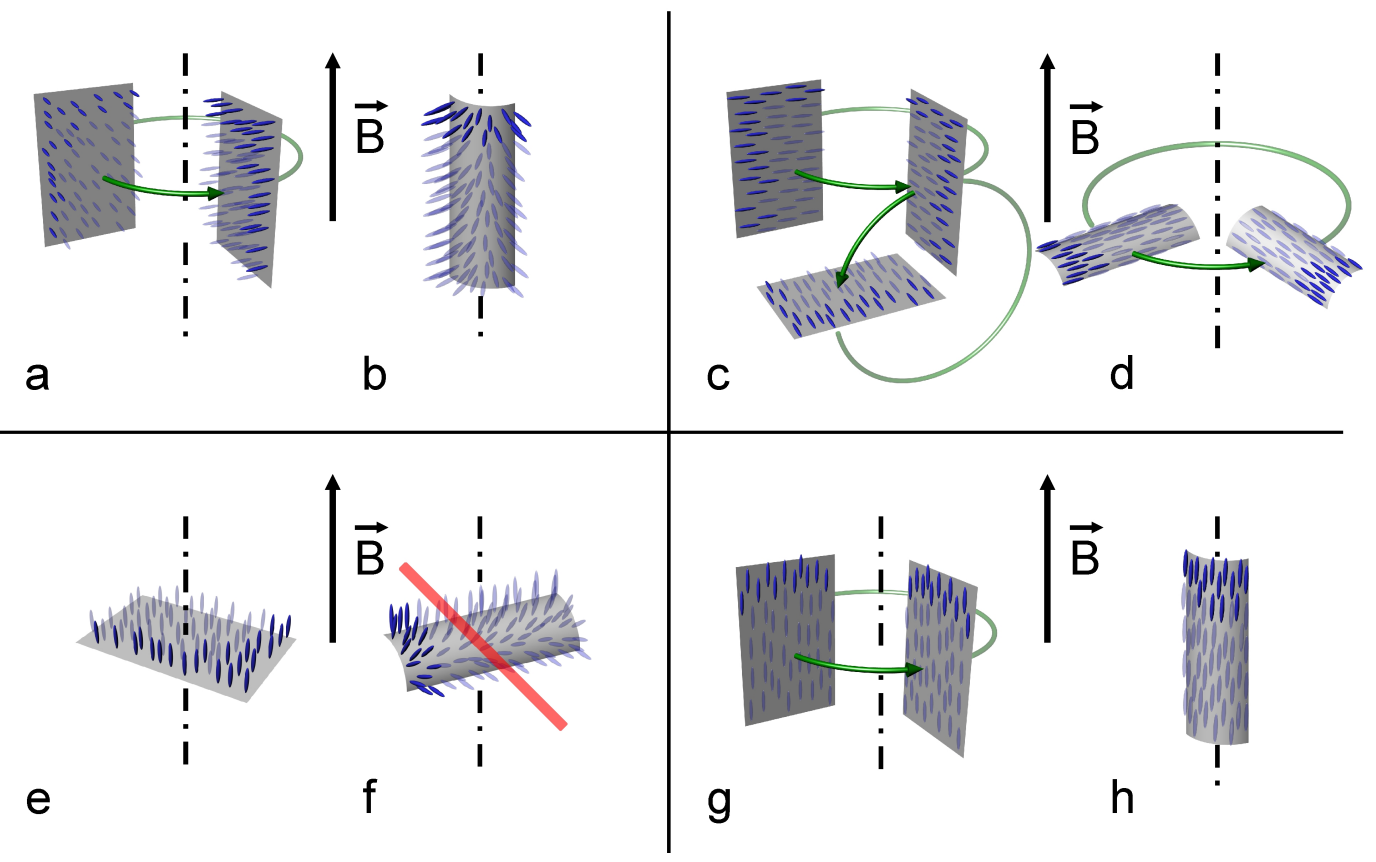

Fig. 1 Alignment of lamellar and cylindrical microstructures is dependent on the anisotropy of magnetic susceptibility and the orientation of the anisotropic units with respect to the inter-material dividing surface (IMDS), shown as the grey surface. Anisotropic units may be surfactants in lyotropic phases, mesogens in thermotropic LC block copolymers, or crystalline domains in semicrystalline block copolymers, for example. They are shown schematically here as rigid cigar-shaped objects in blue. Top row: $\Delta \chi<0$; Bottom row: $\Delta \chi>0$; Left side: Homeotropic or perpendicular anchoring; Right side: Homogeneous or planar anchoring. Alignments which satisfy both the orientational relationship of the anisotropic unit at the IMDS and the magnetic anisotropy are unique only for lamellae in $\mathbf{e}$ and for cylinders in $\mathbf{b}$ and $\mathbf{h}$. All other arrangements are degenerate, as shown by the green arrows which indicate rotations of the structures which are all compatible with the anisotropy and anchoring constraints. f: No orientation of a singlycurved surface with perpendicularly attached units of positive anisotropy can satisfy the constraint that all such units align with the field. A red line has been drawn through this scheme to indicate its aphysical nature.

We hypothesize that field (or sample) rotation may be used to break the degeneracy described above. Our scheme is presented in Figure Fig. 2. The negative anisotropy of the alkyl chains of the surfactant combined with their perpendicular orientation to the IMDS implies that the lamellae align with their surface normals perpendicular to the field direction. Since the IMDS is flat, this produces a degenerate set of lamellae with normals confined in the plane perpendicular to the field direction. For example, as in Case I in Figure Fig. 2, with a field $\mathbf{B}_{x}$, lamellar normals are confined to the $y z$ plane, a condition designated as $\mathbf{n}_{y z}$. If the field direction was instead along the z-axis, $\mathbf{B}_{z}$, the degenerate set would have surface normals confined to the $x y$ plane. Simple rotation of the field (or sample) through 
90 degrees between these two situations then implies that only the set of lamellae with their surface normals along the $\mathrm{y}$-axis, $\mathbf{n}_{y}$, will be stable under both field directions. This defines a unique subset of orientations and in this way, the degeneracy of lamellar alignment under a uniaxial external field is broken. Repeated 90 degree rotations between the two states can be used to further enrich the population of lamellae in the subset, although the effect is nearly complete after only one rotation cycle, as we show. The alignment of hexagonally packed cylindrical microdomains in lyotropic systems is non-degenerate, as described in Figure Fig. 1.

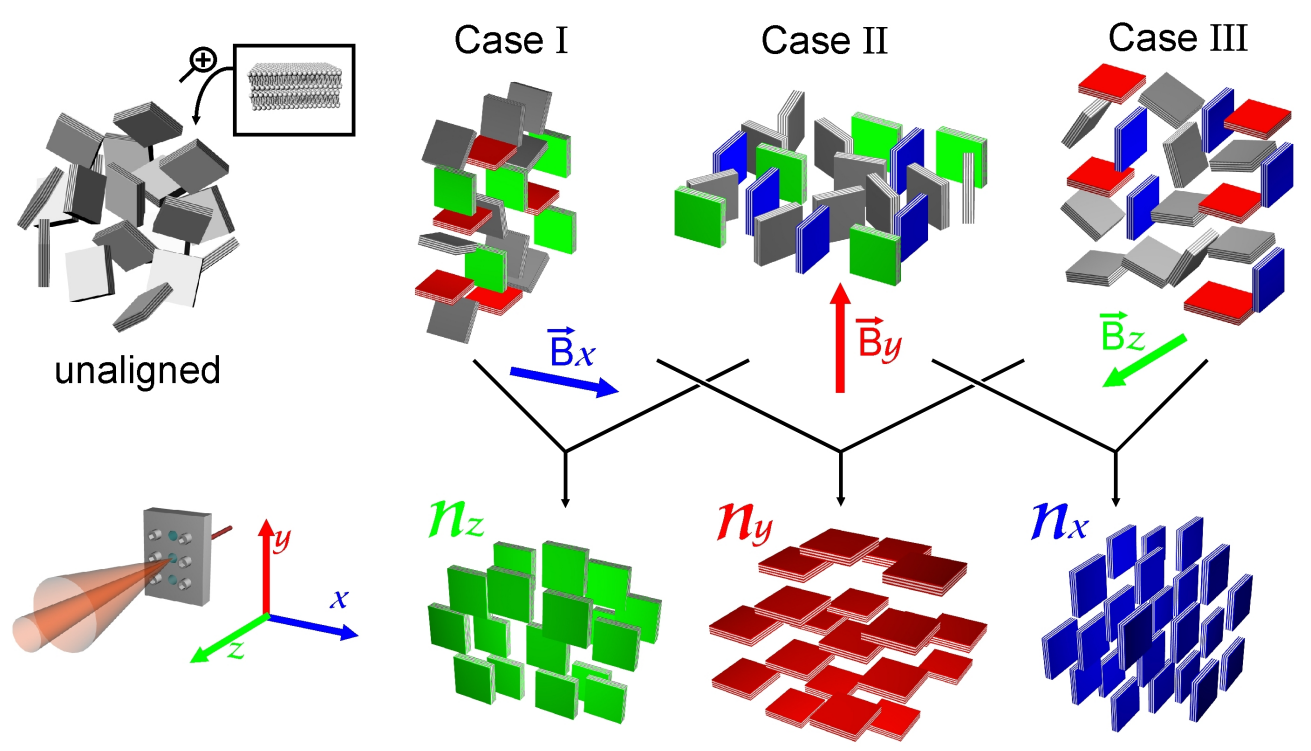

Fig. 2 Magnetic alignment of lyotropic lamellar mesophases produces degenerate sets of lamellae where the lamellar normals lie in the plane orthogonal to the field direction. Case I: $\mathbf{B}_{x}, \mathbf{n}_{y z}$. Case II: $\mathbf{B}_{y}, \mathbf{n}_{x z}$. Case III: $\mathbf{B}_{z}, \mathbf{n}_{x y}$. Lamellae that are aligned along the principal axes, $\mathbf{n}_{x}, \mathbf{n}_{y}$ and $\mathbf{n}_{z}$ are shown in blue, red and green respectively while others are shown in grey. Rotation of the field between two orthogonal directions breaks the degeneracy by selecting the unique subset of lamellae which are compatible with both field directions. For example, $\mathbf{B}_{x y}$, the rotation of the field in the $\mathrm{x}-\mathrm{y}$ plane produces only $\mathbf{n}_{z}$ lamellae, as shown. Likewise $\mathbf{B}_{x z}$ produces $\mathbf{n}_{y}$ and $\mathbf{B}_{y z}$ yields $\mathbf{n}_{x}$.

We have examined lamellar and hexagonal phases formed by poly(oxyethylene) n-alkyl ether materials $C_{12} E_{4}$ and $C_{12} E_{9}$ respectively, where the subscripts refer to the number of carbon atoms and ethylene oxide units in the surfactant. Materials were obtained from Sigma-Aldrich and used as received. The surfactants were combined with ultra-pure water (Millipore Milli-Q) to yield $70 \%$ wt./wt. $\mathrm{C}_{12} \mathrm{E}_{4}$ and $52 \% \mathrm{C}_{12} \mathrm{E}_{9}$ respectively. After homogenization by vortexing at $70{ }^{\circ} \mathrm{C}$ the samples were allowed to cool to room temperature, centrifuged to remove trapped air bubbles and allowed to equilibrate in sealed vials at ambient conditions for 12 hours. The phase behavior was characterized by polarized optical microscopy (POM) and small angle X-ray scattering (SAXS) as described in the Supplementary Information. Field alignment was performed using a $5 \mathrm{~T}$ static magnetic field produced by a superconducting magnet (AMI American Magnetics, Inc.) with split-coil geometry that facilitated sample rotation. A common sample holder was used for both field alignment and SAXS measurements, providing reproducible positioning for scattering after 
each annealing in the magnet. The SAXS patterns of the samples referred herein as "unaligned" were collected using samples which were heated to $70{ }^{\circ} \mathrm{C}$ and cooled back to room temperature in the absence of the magnetic field.

Many non-ionic surfactants exhibit temperature as well as composition dependent order-order transitions between lamellar, cubic and hexagonal phases. In order to unambiguously relate aligned structures examined at room temperature to alignments conducted at elevated temperatures, we first ensure that the temperature ramps used in the field alignment do not involve transitions to alternate ordered phases for the samples studied. The compositions chosen featured direct transitions from a high temperature disordered micellar phase into the hexagonal and lamellar phases on cooling. Temperature dependent small angle x-ray scattering (SAXS) and polarized optical microscopy were used to locate the order-disorder transitions of both materials, $T_{O D T}$. From these data (see Supplementary Information), $C_{12} E_{9}$ at a weight fraction $\varphi=0.52$ exhibits a transition on heating from the HEX phase $\left(H_{1}\right)$ to a disordered micellar phase $\left(L_{1}\right)$ at $47{ }^{\circ} \mathrm{C}$. For $C_{12} E_{4}$ at $\varphi=0.70$, there is a similar transition on heating from the LAM phase $\left(L_{\alpha}\right)$ to a disordered micellar phase $\left(L_{2}\right)$ at $64{ }^{\circ} \mathrm{C}$. Both transitions were observed to be reversible and free of thermal hysteresis. Additionally, both systems were non-crystalline at room temperature as the solidification line is located at sub-ambient temperatures.

Experiments were conducted by placing the sample holder in different orientations with respect to a fixed magnetic field produced by our instrument. SAXS was performed in a fixed geometry, with x-rays incident normal to the plane of the sample. In order to describe the alignment and scattering geometries unambiguously, we represent here the sample and plane of the scattering vector $\mathbf{q}_{x y}$ as fixed, and the field directions as varying. We track the integrated intensity over a q-range covering the (001) reflection, $I_{(001)}$ (arbitrary units, counts per second), which is proportional to the number of scattering elements in the sample. We also compute the second coefficient of the orientational distribution function, $P_{2}$, or the orientation order parameter (described in Supplementary Information) which enables quantification of the degree of alignment. The initially unaligned $L_{\alpha}$ mesophase has $I_{(001)}=0.015$ and $P_{2}=0.01$. Samples were aligned under a field of $\mathrm{B}=5 \mathrm{~T}$ by slowly cooling from the disordered micellar regime $\left(68^{\circ} \mathrm{C}\right)$ to $25^{\circ} \mathrm{C}$ over the course of $\approx 2$ hours. Alignment with the field parallel to the sample plane, (Case I, $\mathbf{B}_{x}$ ) produces a two-fold orientation of the (001) peak of the lamellar microstructure in SAXS, Figure Fig. 3b with $I_{(001)}=0.012$ and $P_{2}=0.74$. Samples aligned from the disordered micellar state with the field along the z-axis (Case III, $\mathbf{B}_{z}$ ), show a more polydomain type scattering and a markedly higher intensity of the (001) peak, Fig. 3c, with $I_{(001)}=0.11$ and $P_{2}=0.73$. These data confirm the scenario depicted in Figure Fig. 2. In Case I, lamellae are aligned with their normals in the $y z$ plane, but with x-rays incident along the $z$-axis, only $\mathbf{n}_{y}$ lamellae are in the Bragg condition. The great majority of aligned lamellae are not in the Bragg condition and do not contribute to the scattered intensity which thus remains small, similar to that of the unaligned sample. By contrast, in Case III, any lamellae which align with the field do so with their normals in the $x y$ plane $\left(\mathbf{n}_{x y}\right)$ and are thus all in the Bragg condition for our scattering geometry, $\mathbf{q}_{x y}$. The resulting scattered intensity is significantly higher than for Case I. This intensity represents a limiting case as all lamellae aligned over the timescale 
of the experiment contribute to scattering in $I_{(001)}$.

Disparities in scattered intensity combined with the order parameter calculation provide quantitative measures which characterize the breaking of degeneracy in the rotational annealing experiment. Alignment after sample rotation is conducted at a temperature below the order-disorder transition temperature of the system. This ensures that lamellae are only re-oriented, as opposed to being freshly aligned on formation from the high temperature disordered micellar phase. Rotation of the sample from Case I to Case III geometry $\left(\mathbf{B}_{x}\right.$ to $\left.\mathbf{B}_{z}\right)$ and re-applying the field should produce no meaningful increase in the scattered intensity compared with alignment produced in a single $\mathbf{B}_{z}$ field application in which, as described, a maximal number of aligned lamellae are in the Bragg condition. The order parameter is also not entirely diagnostic, given the polydomain structure produced by an initial $\mathbf{B}_{z}$ process. Rotation from $\mathbf{B}_{x}$ to $\mathbf{B}_{z}$ and back to $\mathbf{B}_{x}$ however is different. If the system remains degenerate, $I_{(001)}$ should fall from the limiting case represented by a $\mathbf{B}_{z}$ alignment as lamellae fall out of the Bragg condition under the final $\mathbf{B}_{x}$ step. Finally, rotation from Case I to Case II, $\mathbf{B}_{x}$ to $\mathbf{B}_{y}$ should produce little to no scattered intensity in $\mathbf{q}_{x y}$ as all aligned lamellae, with normals along the z-axis $\left(\mathbf{n}_{z}\right)$, would be uniquely out of the Bragg condition if the degeneracy of the system was successfully broken. These expectations are precisely what is observed. For the $\mathbf{B}_{x}-\mathbf{B}_{z}$ rotation, Figure Fig. $3 \mathrm{~d}, I_{(001)}=0.10$ and $P_{2}=0.78$. By contrast, for $\mathbf{B}_{x}-\mathbf{B}_{z}-\mathbf{B}_{x}$, Figure Fig. 3e, $I_{(001)}=0.11$ and $P_{2}=0$.98. Finally, for $\mathbf{B}_{x}-\mathbf{B}_{y}$ rotation, Figure Fig. 3f, $I_{(001)}$ $=0.003$ and $P_{2}=0.21$. The data clearly demonstrate that degeneracy of the system is effectively broken by rotation, resulting in high orientational order, as shown by the azimuthal intensity traces of Figure Fig. 4. The footprint of the $\mathrm{x}$-ray beam is roughly $0.5 \mathrm{~mm}^{2}$. In all cases, except for the polydomain scattering encountered for $\mathbf{B}_{z}$, alignments were consistent over the entire sample area $(\approx 1 \mathrm{~mm} \times 3 \mathrm{~mm})$, as determined by stepping the beam in $0.5 \mathrm{~mm}$ increments across the sample.

Alignment of the $H_{1}$ phase of $C_{12} E_{9}$ was similarly conducted by slowly cooling the sample from the disordered micellar regime at $60{ }^{\circ} \mathrm{C}$ to $25^{\circ} \mathrm{C}$ over 2 hours. The data for Case II and Case III geometries are shown in Figure Fig. 5, showing polydomain 6-fold and 2-fold patterns, consistent with alignment of the long axes of the hexagonally packed cylinders along the field direction. An order parameter of $P_{2}=0.83$ is determined for the Case II alignment, representing a significant increase from the initial unaligned state where $P_{2}$ was 0.09 . Because of the negative diamagnetic anisotropy of the surfactant and its perpendicular orientation to the IMDS, as discussed in Figure Fig. 1, the alignment here is already non-degenerate. However, the effect of the field does not eliminate grain boundaries due to different orientations of hexagonal packing of cylinders in the plane perpendicular to the cylinder long axis. This is why the SAXS of Figure Fig. 5c shows a polydomain pattern. In another system, either a different molecular diamagnetic anisotropy or a different anchoring condition of the anisotropic units at the IMDS can give rise to degenerate alignments with the cylinder long axes perpendicular to the field direction. For example, this has been observed in a liquid crystalline diblock copolymer based on biphenyl benzoate mesogens. ${ }^{23}$ Rotational annealing as described here can be used to impart non-degenerate alignment in such a system. 


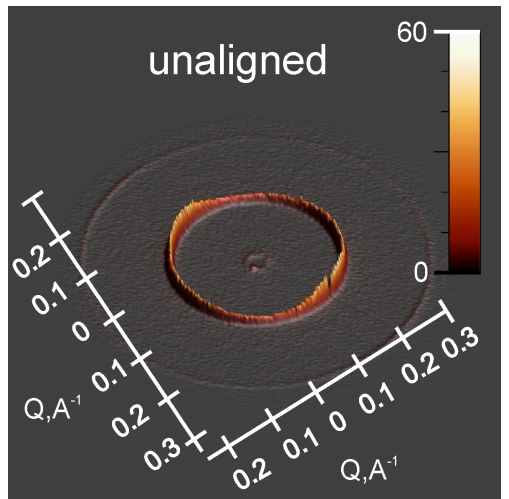

a

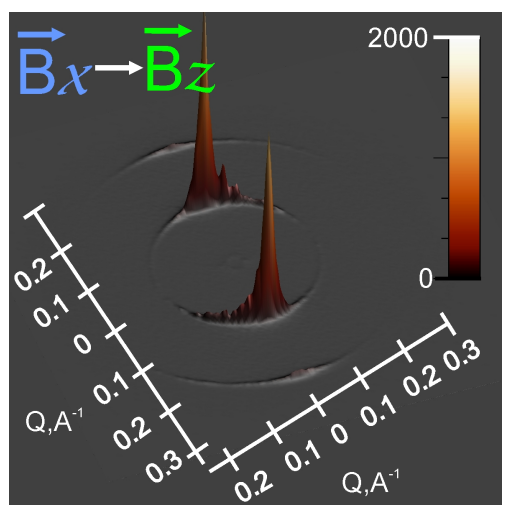

d

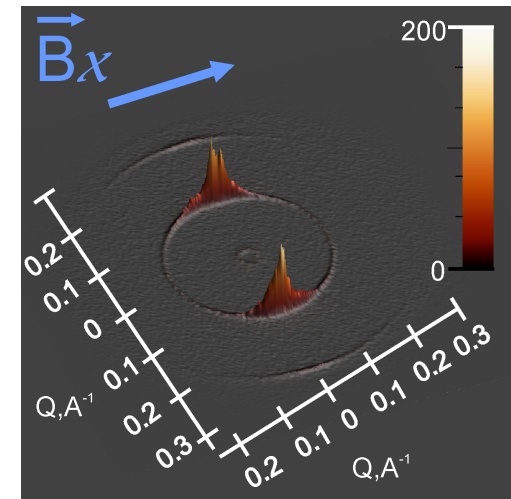

b

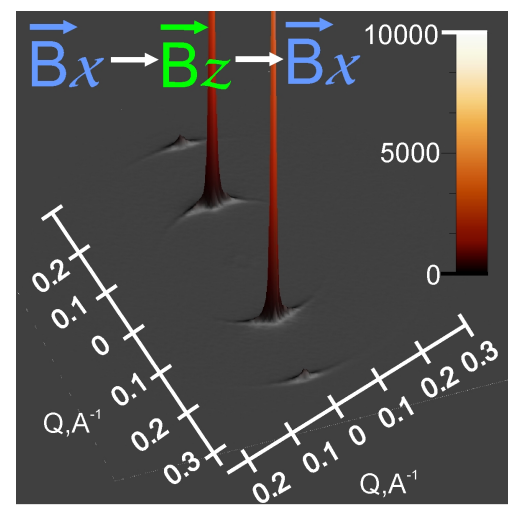

e

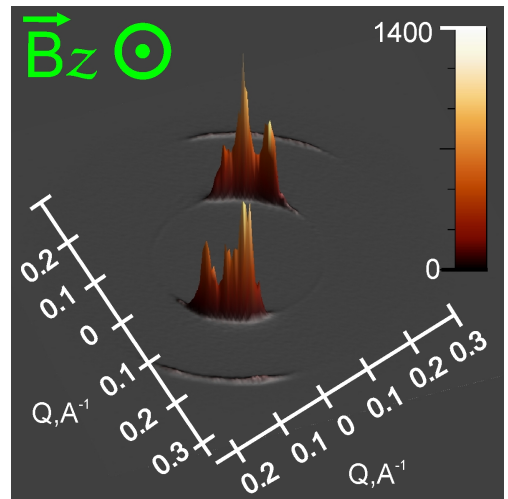

c

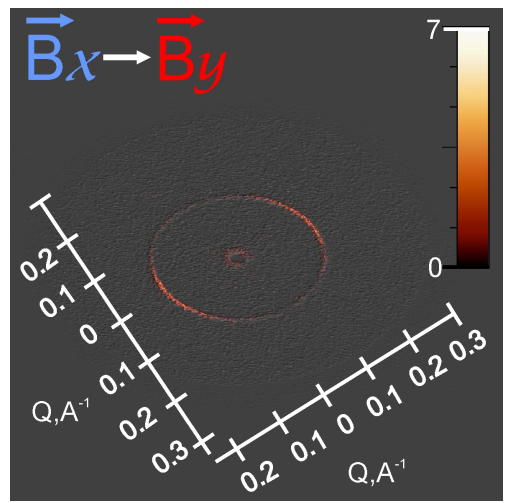

f

Fig. 3 SAXS data from lamellar mesophases. 2-dimensional images were rendered using software as described in ref. ${ }^{29}$ a Initial unaligned material. $I_{(001)}=0.015$ and $P_{2}=0.01$. b Sample after $\mathbf{B}_{x}$ alignment. $I_{(001)}=0.012$ and $P_{2}=$ 0.74. c Sample after $\mathbf{B}_{z}$ alignment. $I_{(001)}=0.11$ and $P_{2}=0.73$. d Alignment under $\mathbf{B}_{x}$ followed by $\mathbf{B}_{z} \cdot I_{(001)}=0.10$ and $P_{2}=0.78$. e Rotation from $\mathbf{B}_{x}$ to $\mathbf{B}_{z}$ and back to $\mathbf{B}_{x} . I_{(001)}=0.11$ and $P_{2}=0.98$. $\mathbf{f}$ Alignment under $\mathbf{B}_{x}$ followed by $\mathbf{B}_{y} . I_{(001)}=0.003$ and $P_{2}=0.21$.

In summary, we have demonstrated that high magnetic fields may be successfully used to orient non-ionic surfactant mesophases and have developed a simple rotational annealing scheme which enables the realization of nondegenerate alignments. We have demonstrated its application here for the production of highly ordered lamellar phases. The alignments achieved were uniform over the length scale of the samples $(\approx 3 \mathrm{~mm})$ and were produced over reasonable timescales of 2 hours. Our method enables the formation of highly aligned lyotropic lamellae in thin films with the lamellar normal uniquely confined along an arbitrary choice of the x, y or z-axes by II-III, I-III or I-II rotations, respectively. The approach is quite general and may be applied to the alignment of degenerate cylindrical systems as well. An improvement to the current scheme would incorporate the ability to continuously rotate samples in the field, with time dependent velocity. Recent work has suggested this as a route to achieving alignment of the weak susceptibility axis in diamagnetic materials. ${ }^{30}$ The $C_{n} E_{m}$ system studied here and other non-ionic mesophases provide excellent templates for nanomaterials synthesis and sequestration. Combined with the demonstrated facile and specific alignment of mesoscale structures, these systems offer an attractive route towards new functional materials. 


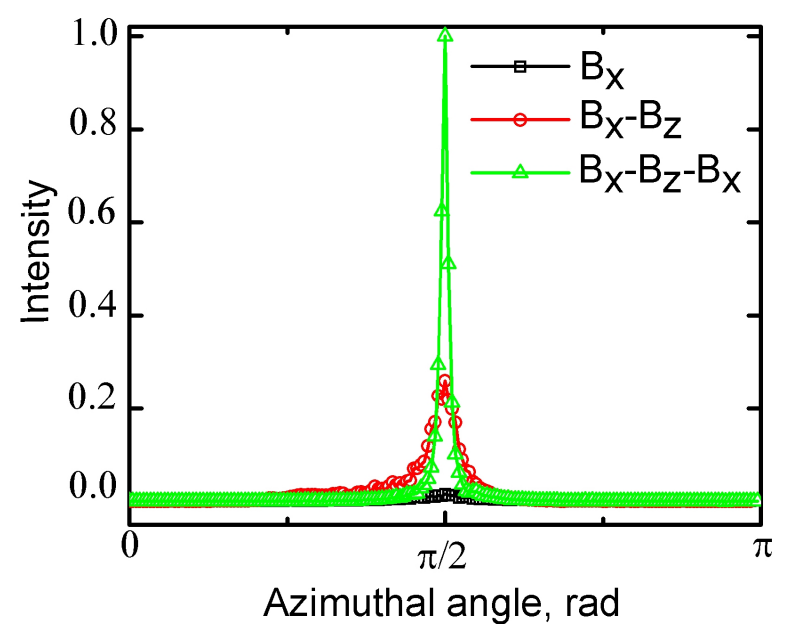

Fig. 4 Normalized azimuthal intensity scans from SAXS data for samples shown in Figure Fig. 3b, d and e.

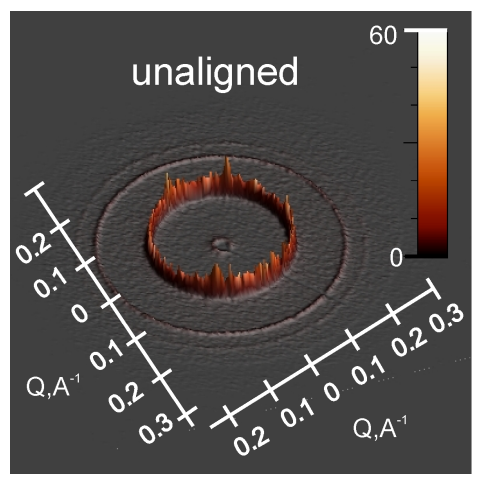

a

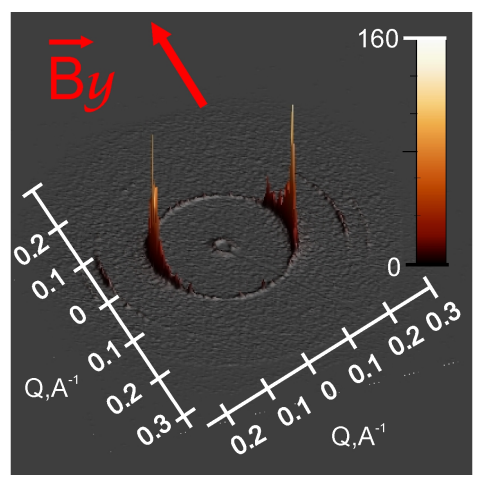

b

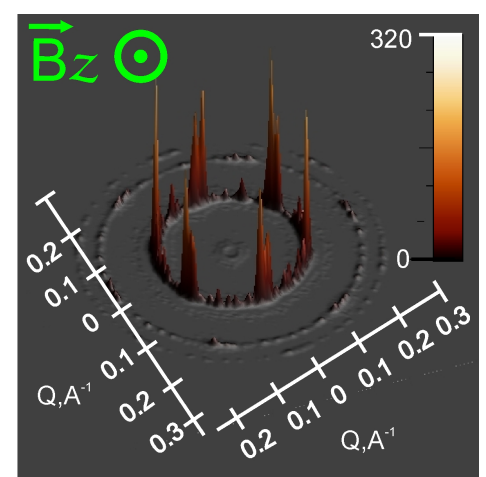

c

Fig. 5 SAXS data from hexagonal mesophases. From left to right: a Initial unaligned material. $I_{(10 \overline{1} 0)}=0.008$ and $P_{2}$ $=0.09$. b Sample after $\mathbf{B}_{y}$ alignment. $I_{(10 \overline{1})}=0.01$ and $P_{2}=0.83$. $\mathbf{c}$ Sample after $\mathbf{B}_{z}$ alignment produces a polydomain scattering due to the presence of different hexagonal grain orientations in the plane normal to the field direction. 


\section{References}

[1] S. Sun, Solar energy materials and solar cells, 2003, 79, 257-264.

[2] C. Osuji, C. Chao, I. Bita, C. Ober and E. Thomas, Adv. Funct. Mater., 2002, 12, 753-758.

[3] S. Valkama, H. Kosonen, J. Ruokolainen, T. Haatainen, M. Torkkeli, R. Serimaa, G. Ten Brinke and O. Ikkala, Nat. Mater., 2004, 3, 872-876.

[4] A. Edrington, A. Urbas, P. DeRege, C. Chen, T. Swager, N. Hadjichristidis, M. Xenidou, L. Fetters, J. Joannopoulos, Y. Fink et al., Adv. Mater., 2001, 13, 421-425.

[5] I. Bita, J. Yang, Y. Jung, C. Ross, E. Thomas and K. Berggren, Science, 2008, 321, 939.

[6] S. Park, D. Lee, J. Xu, B. Kim, S. Hong, U. Jeong, T. Xu and T. Russell, Science, 2009, 323, 1030.

[7] T. Thurn-Albrecht, J. Schotter, G. Kastle, N. Emley, T. Shibauchi, L. Krusin-Elbaum, K. Guarini, C. Black, M. Tuominen and T. Russell, Science, 2000, 290, 2126.

[8] I. Hamley, Angew. Chem. Int. Ed., 2003, 42, 1692-1712.

[9] R. Hayward, P. Alberius, E. Kramer and B. Chmelka, Langmuir, 2004, 20, 5998-6004.

[10] S. Darling, Prog. Polym. Sci., 2007, 32, 1152-1204.

[11] T. Morkved, M. Lu, A. Urbas, E. Ehrichs, H. Jaeger, P. Mansky and T. Russell, Science, 1996, $273,931$.

[12] T. Thurn-Albrecht, J. DeRouchey, T. Russell and H. Jaeger, Macromolecules, 2000, 33, 3250-3253.

[13] A. Böker, A. Knoll, H. Elbs, V. Abetz, A. Müller and G. Krausch, Macromolecules, 2002, 35, 1319-1325.

[14] C. Chao, X. Li, C. Ober, C. Osuji and E. Thomas, Adv. Funct. Mater., 2004, 14, 364-370.

[15] A. Firouzi, D. Schaefer, S. Tolbert, G. Stucky and B. Chmelka, J. Am. Chem. Soc., 1997, 119, 9466-9477.

[16] A. Rapp, K. Ermolaev and B. M. Fung, J. Phys. Chem. B, 1999, 103, 1705-1711.

[17] J. Clawson, G. Holland and T. Alam, PCCP, 2006, 8, 2635-2641.

[18] J. Lagerwall, G. Scalia, M. Haluska, U. Dettlaff-Weglikowska, S. Roth and F. Giesselmann, Adv. Mater., 2007, 19, 359-359.

[19] Y. Yamauchi, M. Sawada, M. Komatsu, A. Sugiyama, T. Osaka, N. Hirota, Y. Sakka and K. Kuroda, Chem. Asian J., 2007, 2, 1505-1512. 
[20] J. W. Emsley, J. E. Long, G. R. Luckhurst and P. Pedrielli, Phys. Rev. E, 1999, 60, 1831-1839.

[21] B. Pate, S. Choi, U. Werner-Zwanziger, D. Baxter, J. Zaleski and M. Chisholm, Chem. Mater., 2002, 14, 19301936.

[22] I. Hamley, V. Castelletto, Z. Lu, C. Imrie, T. Itoh and M. Al-Hussein, Macromolecules, 2004, 37, 4798-4807.

[23] C. Osuji, P. Ferreira, G. Mao, C. Ober, J. Vander Sande and E. Thomas, Macromolecules, 2004, 37, 9903-9908.

[24] M. Adachi, F. Takazawa, N. Tomikawa, M. Tokita and J. Watanabe, Polymer Journal, 2007, 39, $155-162$.

[25] Y. Tao, H. Zohar, B. Olsen and R. Segalman, Nano Lett., 2007, 7, 2742-2746.

[26] T. Grigorova, S. Pispas, N. Hadjichristidis and T. Thurn-Albrecht, Macromolecules, 2005, 38, 7430-7433.

[27] C. Osuji, J. Chen, G. Mao, C. Ober and E. Thomas, Polymer, 2000, 41, 8897-8907.

[28] H. Shao, H. Gang and E. Sirota, Phys. Rev. E., 1998, 57, 6265-6268.

[29] I. Horcas, R. Fernandez, J. Gomez-Rodriguez, J. Colchero, J. Gomez-Herrero and A. Baro, Rev. Sci. Instrum., 2007, 78, 013705 .

[30] T. Kimura and M. Yoshino, Langmuir, 2005, 21, 4805-4808.

\section{Acknowledgements}

The authors are grateful for experimental assistance provided by Manesh Gopinadhan. This work was partially funded by the Yale Institute for Nanoscience and Quantum Engineering (YINQE). 